\title{
Obstructive sleep apnoea in patients with fibrotic diffuse parenchymal lung disease-characterization and treatment compliance assessment
}

\author{
Ana Verónica Cardoso, $M D^{1}$, Nuno Pereira, $M D^{2}$, Inês Neves, $M D^{3}$, Vanessa Santos, $M D^{1}$, José Miguel Jesus, $M D^{4}$, \\ Natália Melo, $\mathrm{MD}^{1}$, Patrícia Caetano Mota, $\mathrm{MD}^{1,2}$, António Morais, $\mathrm{PhD}^{1,2}$, Marta Drummond, $\mathrm{PhD}^{1,2}$
}

\begin{abstract}
AV Cardoso, N Pereira, I Neves, V Santos, JM Jesus, N Melo, PC Mota, A Morais, M Drummond. Obstructive sleep apnoea in patients with fibrotic diffuse parenchymal lung disease-characterization and treatment compliance assessment. Can J Respir Ther 2018;54(2):35-40. doi: 10.29390/cjrt-2018-005.
\end{abstract}

\begin{abstract}
Introduction: Recent studies have reported a high prevalence of obstructive sleep apnoea (OSA) among patients with diffuse parenchymal lung disease (DPLD), mainly idiopathic pulmonary fibrosis (IPF). Effective OSA treatment appears to have a positive impact on morbidity and mortality in these patients. However, poor compliance to positive airway pressure (PAP) treatment in fibrotic DPLD patients has been reported. The aims of our study were to characterize patients with fibrotic DPLD and OSA and to assess their compliance to PAP treatment.

Methods: Forty-nine patients with fibrotic DPLD underwent level III polysomnography. Auto-adjusting PAP (APAP) treatment was proposed for those patients with moderate-to-severe OSA and those with mild OSA with daytime sleepiness and/or cardiovascular disease. The APAP treatment compliance was assessed after 1 month of therapy.

Results: The distribution of the 49 fibrotic DPLD patients included was as follows: 21 with chronic hypersensitivity pneumonitis, 12 with IPF, 10 with connective-tissue associated DPLD, 4 with stage IV sarcoidosis, 1 with idiopathic pleuropulmonary fibroelastosis, and 1 with DPLD-associated vasculitis. Thirty-four (69.4\%) of the patients presented with OSA; 22 had mild OSA, and 12 had moderate-to-severe OSA. APAP treatment was prescribed in 17 of the patients. After 1 month of therapy, all patients used APAP more than 70\% of the nights for more than $4 \mathrm{~h}$ per night.

Conclusion: We found a high prevalence of OSA among all of the patients with fibrotic DPLD (not only IPF). Despite certain difficulties, it was possible to achieve good APAP compliance in these patients.
\end{abstract}

Key Words: obstructive sleep apnea; diffuse parenchymal lung disease; positive airway pressure treatment; compliance

\begin{abstract}
INTRODUCTION
Diffuse parenchymal lung diseases (DPLDs) are a heterogeneous group of disorders resulting from damage to the lung parenchyma by varying patterns of inflammation and fibrosis [1,2]. Fibrotic DPLD, namely idiopathic pulmonary fibrosis (IPF), is associated with high morbidity and mortality. Frequently, these patients have exercise intolerance and hypoxemia that can progress to respiratory failure, pulmonary hypertension, and death [3].

Recently published studies [4-10] have reported a 59\%-90.3\% prevalence of obstructive sleep apnoea (OSA) among patients with DPLD, mainly IPF. In these patients, OSA exhibits atypical features, such as a lack of association between severity of OSA and body mass index (BMI) or daytime sleepiness $[4,5]$. The mechanism underlying OSA and fibrotic DPLD linkage is probably related to the decreased lung volumes in these diseases, which can reduce the upper airway stability and increase resistance due to decreased traction in the upper airway. These changes can facilitate upper airway collapse, especially during REM sleep, when the functional residual capacity is further reduced due to intercostal muscle inactivity [11-14]. Although the majority of studies regarding the mechanism causing OSA included only patients with IPF, we hypothesize that all patients with fibrotic DPLD have an increased risk for this sleep breathing disorder (SBD). In these patients, OSA may be one reason for their impaired sleep quality $[4,5]$ and, consequently,
\end{abstract}

may have a negative influence on daily activities and overall quality of life [15-17]. In addition, underlying OSA and other SBDs, such as sleep oxygen desaturation, appear to have a negative influence on morbidity and mortality in DPLD patients [7, 8].

The timely diagnosis and management of OSA in DPLD patients may be an intervention that can significantly improve these patients' outcomes $[8,18]$. The main issue in sleep medicine is not only the recognition of sleep disorders but also providing effective treatment. There have been few studies addressing OSA treatment in DPLD patients, and all of them included only IPF patients $[8,19,20]$. That population is associated with a high incidence of positive airway pressure (PAP) nonacceptance or poor compliance, which can only be eliminated through an intense follow-up by a well-organized sleep centre $[19,20]$. However, recent studies have shown that effective PAP treatment in IPF patients with OSA resulted in a significant improvement in daily living activities based on a functional outcomes of sleep questionnaire [8, 19] as well as quality of sleep and life [8]. PAP treatment may also influence the mortality in these patients [8] despite the high incidence of nonacceptance and poor compliance to PAP in IPF patients with OSA $[8,19,20]$.

Based on the abovementioned information, the aims of our study were to characterize patients with concomitant fibrotic DPLD and OSA and to assess their compliance to PAP treatment.

\footnotetext{
${ }^{1}$ Pulmonology Department, Centro Hospitalar São João, Oporto, Portugal

${ }^{2}$ Faculty of Medicine of Porto University, Oporto, Portugal

${ }^{3}$ Pulmonology Department, Unidade Local de Saúde de Matosinhos, Oporto, Portugal

${ }^{4}$ Radiology Department, Centro Hospitalar de São João, Oporto, Portugal

Correspondence: Ana Verónica Cardoso, Pulmonology Department, Centro Hospitalar de São João, Alameda Prof. Hernâni Monteiro, $4200-319$ Oporto,

Portugal. Tel.: +351 938847996; E-mail:car_veronica@sapo.pt
} 


\section{Subjects}

This prospective study was conducted at the Pulmonology Department of the Centro Hospitalar São João in Oporto (Portugal) from July 2015 to March 2017. Patients who had been followed-up at the Diffuse Lung Disease Outpatient Clinic of the Pulmonology Department were assessed. All of the patients were informed about the study and voluntarily signed their informed consent. This study was approved by the hospital ethics committee.

The inclusion criteria were as follows: diagnosis of fibrotic DPLD namely IPF, usual interstitial pneumonia (UIP)-like fibrosis, fibrotic nonspecific interstitial pneumonia (NSIP) (idiopathic or NSIP-like), idiopathic pleuropulmonary fibroelastosis, or stage IV sarcoidosis. In addition, they had to exhibit a stable disease; that is, no treatment change and no deterioration in the forced vital capacity (FVC) $>10 \%$ and/or diffusing capacity of the lungs for carbon monoxide (DLCO) $>15 \%$ for the last 3 months.

The exclusion criteria were as follows: known risk factors for sleep disorders (BMI $\geq 30 \mathrm{~kg} / \mathrm{m}^{2}$, cranial-facial deformities, and benzodiazepine use), neuromuscular diseases, severe psychiatric disorders, other chronic pulmonary diseases, a partial pressure of $\mathrm{O}_{2}$ in the arterial blood $\left(\mathrm{PaO}_{2}\right)<60 \mathrm{mmHg}$ at rest on room air, and previously diagnosed OSA.

Of the 65 eligible patients, 49 agreed to participate in the study. They underwent level III polysomnography (PSG III) and were asked to fill out the Epworth Sleepiness Scale (ESS). Each patient's epidemiological data, co-morbidities, pulmonary function test (PFT), 6-min walk test (6MWT), blood gas analysis, fibrosis score, and echocardiogram results were retrieved, retrospectively, from their hospital records. All tests and assessments were carried out within a maximum period of 2 months before inclusion in the study and the accomplishment of the respective polysomnography.

\section{Polysomnography}

The diagnosis of OSA was based on a six-channel overnight PSG III study (ResMed® Embletta ${ }^{\mathrm{TM}}$ Gold Portable Testing Device; Embla, Broomfield, CO, USA), which included monitoring of the heart rate, oronasal airflow (using a thermistor and nasal cannula), respiratory effort (using abdominal and thoracic strain gauges), oxygen saturation (using pulse oximetry), snoring, and body position. All of the sleep studies were manually scored by experienced sleep technicians, and apnoea and hypopnoea were defined according to the criteria of American Academy of Sleep Medicine (AASM) [21, 22]. Apnoea was defined as the reduction in airflow $\geq 90 \%$ of baseline, lasting $\geq 10 \mathrm{~s}$, and hypopnoea as the reduction in baseline airflow $\geq 30 \%$ with $\geq 3 \%$ desaturation, lasting $\geq 10 \mathrm{~s}$. The apnoea-hypopnoea index (AHI) was calculated as the number of apnoea and hypopnoea events per hour of sleep. OSA severity is defined as mild for $\mathrm{AHI} \geq 5$ and $<15$, moderate for $\mathrm{AHI} \geq 15$ and $\leq 30$, and severe for AHI > 30/h [21]. The oxygen desaturation index (ODI), defined as the number of arterial oxygen desaturations per hour $\geq 3 \%$ [21], was also reported. We considered desaturation significant when ODI $>10 / \mathrm{h}$ and/or sleep time with $\mathrm{SpO}_{2}$ less than $90 \%$ (ST<90\%) was more than 20\% [23]. Patients with OSA and ST<90\% scores greater than $20 \%$ underwent nocturnal oximetry after beginning OSA treatment.

\section{PFT and 6MWT}

The spirometry (forced expiratory volume in the first second (FEV1), forced vital capacity (FVC) and FEV1/FVC ratio), static lung volume measurements (total lung capacity (TLC), residual volume (RV) and RV/ TLC ratio via body box plethysmography) and DLCO via single-breath technique were performed using the Jaeger ${ }^{\circledR}$ MasterScreen Body with the patient in a seated position, according to the approved standards [24].

The 6MWT was performed using the protocol approved by the European Respiratory Society/American Thoracic Society Task Force [25].

\section{Fibrosis score}

The radiological extent of pulmonary fibrosis was evaluated using the same fibrosis score as that used in the composite physiological index validation [26]. All patients underwent high-resolution computed tomography (HRCT), and the images were independently evaluated by two thoracic radiologists (the same two radiologists evaluated all HRCTs) at five levels (origin of great vessels, mid-arch of aorta, carina, pulmonary venous confluence, and $1 \mathrm{~cm}$ above the right dome of diaphragm) for the extent of fibrosis [27]. The alterations were graded as follows: $1=$ predominantly fine intralobular fibrosis, 2 = predominantly microcystic honeycombing (comprised of air spaces less than $4 \mathrm{~mm}$ in diameter), and $3=$ predominantly macrocystic honeycombing (comprised of air spaces more than $4 \mathrm{~mm}$ in diameter), with a maximum score of 15 for five levels [26].

\section{ESS}

The ESS is the most widely used instrument for measuring daytime sleepiness in clinical practice. It is simple, self-administered, and measures the risk of falling asleep in 8 specific situations. A score higher than 11 is associated with subjective daytime sleepiness [28].

\section{APAP treatment and compliance}

OSA treatment, with auto-adjusting continuous positive airway pressure (APAP), was proposed for those patients with moderate-to-severe OSA $(\mathrm{AHI} \geq 15 / \mathrm{h})$, and those with mild OSA with daytime sleepiness and/or cardiovascular disease (including arterial hypertension, diabetes mellitus, coronary disease, or stroke).

In Portugal, the cost of APAP treatment, which includes delivery and technical maintenance by domiciliary respiratory care providers, is completely covered by the Portuguese national health system; therefore, treatment is provided at no additional cost to the patient [29]. In our department, it is a common practice to prescribe, at least initially, APAP to OSA patients instead continuous positive airway pressure (CPAP) because it spares pressure titration.

Prior to APAP treatment, all patients received education from a sleep physician regarding OSA, its known effects on comorbidities, proper sleep hygiene, adjunctive/conservative methods to improve sleep, and the importance of treatment compliance. Each patient was scheduled for a clinical appointment with a sleep physician 1 month after starting APAP therapy. At that point, the clinical symptoms were evaluated as well as APAP compliance variables (percentage of nights of APAP use and number of hours per night), 90\% night-time pressure (P90) and residual AHI which were assessed by analysing the data from equipment's memory card.

The compliance to therapy was classified as good if the patient used APAP more than $70 \%$ of the nights and more than $4 \mathrm{~h} /$ night and poor if the usage was less than $70 \%$ of the nights and/or less than $4 \mathrm{~h} /$ night [30]. During the follow-up of these patients, whenever necessary, heated humidification was added, nasal corticosteroids were prescribed, and masks were changed according to the patient's preference or to control the excessive leaking to improve the efficacy, comfort, and compliance.

\section{Statistical analysis}

The data were described as mean and standard deviation (SD) for quantitative variables, and compared using the Mann-Whitney U or KruskalWallis tests, as appropriate. The categorical variables were described as counts and proportions and compared using $\chi^{2}$ or Fisher's exact tests. The statistical analysis was performed using SPSS version 24.0 software (SPSS Inc., Chicago, Illinois, USA). All of the probabilities were twotailed, and $p$ values $<0.05$ were regarded as significant.

\section{RESULTS}

The study group included 49 patients with diagnosis of chronic hypersensitivity pneumonitis (CHP) (UIP-like or fibrotic NSIP-like, $n=21$ ), IPF $(n=12)$, connective-tissue associated DPLD (UIP-like or fibrotic NSIP-like, $n=10)$, stage IV sarcoidosis $(n=4)$, idiopathic pleuropulmonary fibroelastosis $(n=1)$, and vasculitis associated DPLD (UIP-like, $n=1)$. Demographic and clinical data are shown in Table 1 .

A diagnosis of OSA (AHI $\geq 5$ events/h) was made in 34 (69.4\%) patients, of those $22(64.7 \%)$ had mild OSA and $12(35.3 \%)$ had moderate-to-severe OSA. The prevalence of OSA was $83.3 \%$ in the IPF, $76.2 \%$ in the CHP, $70.0 \%$ in the connective-tissue associated DPLD, and $25.0 \%$ in the stage IV sarcoidosis patients. Neither of the two patients with idiopathic pleuropulmonary fibroelastosis and vasculitisassociated DPLD presented with OSA. 
TABLE 1

Demographic and clinical data of the patients included in this study

\begin{tabular}{|c|c|c|c|}
\hline Variables & All patients $(n=49)$ & Patients with OSA $(n=34)$ & Patients eligible for APAP treatment $(n=25)$ \\
\hline Age (years) & $67.2 \pm 12.2$ & $69.2 \pm 11.2$ & $71.2 \pm 10.0$ \\
\hline Gender (male/female) & $26(53.1) / 23(46.9)$ & $16(47.1) / 18(52.9)$ & $10(40.0) / 15(60.0)$ \\
\hline \multicolumn{4}{|l|}{ Smoking history } \\
\hline Current smokers & $4(8.2)$ & $2(5.9)$ & $1(4.0)$ \\
\hline Ex-smokers & $16(32.7)$ & $10(29.4)$ & $6(24.0)$ \\
\hline Non-smokers & $29(59.2)$ & $22(64.7)$ & $18(72.0)$ \\
\hline \multicolumn{4}{|l|}{ Diffuse parenchymal lung disease } \\
\hline $\mathrm{CHP}$ & $21(42.9)$ & $16(47.1)$ & $13(52.0)$ \\
\hline IPF & $12(24.5)$ & $10(29.4)$ & $6(24.0)$ \\
\hline Connective-tissue associated DPLD & $10(20.4)$ & $7(20.6)$ & $5(20.0)$ \\
\hline Stage IV sarcoidosis & $4(8.2)$ & $1(2.9)$ & $1(4.0)$ \\
\hline Idiopathic pleuropulmonary fibroelastosis & $1(2.0)$ & $0(0)$ & $0(0)$ \\
\hline Vasculitis associated DPLD & $1(2.0)$ & $0(0)$ & $0(0)$ \\
\hline \multicolumn{4}{|l|}{ Comorbidities } \\
\hline GERD & $34(69.4)$ & $21(61.8)$ & $18(72.0)$ \\
\hline Arterial hypertension & $23(46.9)$ & $18(52.9)$ & $15(60.0)$ \\
\hline Diabetes mellitus & $9(18.4)$ & $7(20.6)$ & $7(28.0)$ \\
\hline Coronary disease and/or heart failure & $7(14.3)$ & $5(14.7)$ & $5(20.0)$ \\
\hline Stroke & $4(8.2)$ & $3(8.8)$ & $3(12.0)$ \\
\hline Pulmonary hypertension & $6 / 35(17.1)$ & $4 / 22(18.2)$ & $4 / 22(18.2)$ \\
\hline
\end{tabular}

All data are given as the mean $\pm S D$ or $\mathrm{n}(\%)$. OSA, obstructive sleep apnoea; APAP, auto-adjusting positive airway pressure; CHP, chronic hypersensitivity pneumonitis; IPF, idiopathic pulmonary fibrosis; DPLD, diffuse parenchymal lung disease; GERD, gastro-oesophageal reflux disease.

\section{TABLE 2}

Pulmonary function test results, fibrotic scores, ESS scores, and polysomnographic parameters of the included patients

\begin{tabular}{|c|c|c|c|}
\hline Variables & All patients $(n=49)$ & Patients with OSA $(n=34)$ & Patients eligible for PAP treatment $(n=25)$ \\
\hline $\mathrm{AHI}$ (events/h) & $\begin{array}{l}<5-15 \text { patients } \\
5-14-22 \text { patients } \\
\geq 15-12 \text { patients }\end{array}$ & $\begin{array}{l}5-14-22 \text { patients } \\
\geq 15-12 \text { patients }\end{array}$ & $\begin{array}{l}5-14-13 \text { patients } \\
\geq 15-12 \text { patients }\end{array}$ \\
\hline \multicolumn{4}{|l|}{ Pulmonary function tests } \\
\hline FEV1 (\% predicted) & $89.3 \pm 23.9$ & $94.8 \pm 23.6$ & $96.3 \pm 26.4$ \\
\hline FVC (\% predicted $)$ & $86.1 \pm 18.9$ & $89.8 \pm 18.4$ & $91.2 \pm 20.4$ \\
\hline FEV1/FVC (\%) & $80.3 \pm 9.6$ & $81.6 \pm 9.3$ & $81.1 \pm 10.5$ \\
\hline TLC (\% predicted) & $84.4 \pm 14.8$ & $85.5 \pm 14.5$ & $87.3 \pm 15.3$ \\
\hline DLCO (\% predicted) & $65.8 \pm 18.6$ & $67.5 \pm 18.8$ & $61.3 \pm 15.9$ \\
\hline \multicolumn{4}{|l|}{ Six-minute walk test } \\
\hline Distance (meters) & $405.0 \pm 124.8$ & $401.8 \pm 113.9$ & $373.2 \pm 94.4$ \\
\hline $\mathrm{SpO}$ before $(\%)$ & $95.5 \pm 2.5$ & $95.7 \pm 2.2$ & $95.7 \pm 1.5$ \\
\hline $\mathrm{SpO}_{2}^{2}$ after $(\%)$ & $87.4 \pm 6.1$ & $87.5 \pm 5.8$ & $86.5 \pm 6.4$ \\
\hline \multicolumn{4}{|l|}{ Blood gas analysis } \\
\hline $\mathrm{PaO}_{2}(\mathrm{mmHg})$ & $79.6 \pm 10.7$ & $79.1 \pm 10.9$ & $78.2 \pm 10.5$ \\
\hline $\mathrm{PaCO}_{2}(\mathrm{mmHg})$ & $38.7 \pm 5.1$ & $39.2 \pm 5.3$ & $39.2 \pm 5.9$ \\
\hline Fibrosis score & $9.3 \pm 3.4$ & $8.5 \pm 3.1$ & $8.8 \pm 3.3$ \\
\hline ESS & $6.7 \pm 5.2$ & $6.6 \pm 4.6$ & $8.1 \pm 4.7$ \\
\hline$>11$ & $9(18.4)$ & $5(14.7)$ & $5(20.0)$ \\
\hline$<11$ & $40(81.6)$ & $29(85.3)$ & $20(80.0)$ \\
\hline \multicolumn{4}{|l|}{ Polysomnography III } \\
\hline $\mathrm{AHI}$ (events/h) & $11.3 \pm 9.9$ & $15.0 \pm 9.8$ & $17.1 \pm 10.5$ \\
\hline Apnea index (events/h) & $4.4 \pm 5.1$ & $5.8 \pm 5.5$ & $5.9 \pm 6.3$ \\
\hline Hypopnoea (events/h) & $7.0 \pm 7.3$ & $9.2 \pm 7.8$ & $11.2 \pm 8.0$ \\
\hline ODI (events/h) & $11.5 \pm 10.7$ & $15.2 \pm 11.1$ & $17.5 \pm 11.5$ \\
\hline$>10$ events/h & $23(46.9)$ & $23(67.6)$ & $20(80.0)$ \\
\hline$<10$ events/h & $26(53.1)$ & $11(32.4)$ & $5(20.0)$ \\
\hline Minimum SpO $(\%)$ & $83.0 \pm 6.9$ & $81.5 \pm 7.6$ & $80.1 \pm 7.4$ \\
\hline Average $\mathrm{SpO}_{2}^{2}(\%)$ & $92.9 \pm 2.9$ & $92.7 \pm 3.0$ & $92.1 \pm 3.0$ \\
\hline $\mathrm{ST}<90 \%(\%)$ & $14.4 \pm 24.7$ & $16.9 \pm 27.5$ & $21.9 \pm 30.0$ \\
\hline$>20 \%$ & $12(24.5)$ & $10(29.4)$ & $10(40.0)$ \\
\hline$<20 \%$ & $37(75.5)$ & $24(70.6)$ & $15(60.0)$ \\
\hline
\end{tabular}

All data are given as the mean $\pm S D$ or $\mathrm{n}(\%)$. ESS, Epworth sleepiness score; OSA, obstructive sleep apnoea; PAP, positive airway pressure; AHI, apnoeahypopnoea index; FEV1, forced expiratory volume in 1 second; FVC, forced vital capacity; TLC, total lung capacity; DLCO, diffusing capacity of lung for carbon monoxide; ODI, oxygen desaturation index; $S T<90 \%$, sleep time with $\mathrm{SpO}_{2}$ below $90 \%$.

PFTs and 6MWTs, blood gas analyses, fibrosis scores, ESS scores, and PSG parameters are shown in Table 2. Most respiratory events consisted of hypopnoea rather than apnoea. ODI was higher than 10 events/h in $23(46.5 \%)$ of the patients, all with OSA. The mean of the ODI was statistically different between those patients with and without OSA $(15.2 \pm 11.1$ events $/ \mathrm{h}$ and $3.4 \pm 2.1$ events/h, respectively, 
$p=0.009)$. Twelve patients (24.5\%) exhibited more than $20 \%$ of their ST $<90 \%$, from which 10 (83.3\%) had OSA. Despite the lack of statistical significance, the mean percentage of ST $<90 \%$ was substantially higher in OSA patients than in those without this comorbidity $(16.9 \pm 27.5 \%$ and $8.4 \pm 15.7 \%$, respectively, $p=0.06$ ). There were no significant differences concerning age, gender, smoking history, comorbidities, PFTs, 6MWTs, blood gas analyses, fibrosis scores, and ESS scores between patients with and without OSA (Table 3).

Among OSA patients, 25 were eligible for APAP treatment according to the criteria described above. Of these, 4 patients refused APAP treatment (none of these had excessive daytime sleepiness), 2 patients died before starting treatment, and 2 others reported APAP intolerance due to claustrophobia and/or insomnia and were excluded 1 week after unsuccessful attempts. APAP treatment was prescribed for the remaining patients. Supplemental oxygen was not added to any of these patients.

The APAP treatment compliance after 1 month is described in Table 4. All of the patients used APAP for more than $70 \%$ of the nights and for more than $4 \mathrm{~h}$ /night. Those patients with previous ST<90\% scores greater than $20 \%(n=8)$ underwent nocturnal oximetry while
TABLE 4

APAP compliance of 17 included patients* after 1-month follow-up

\begin{tabular}{lc}
\hline Variables & $\begin{array}{c}\text { Patients under APAP } \\
\text { treatment }(\boldsymbol{n}=\mathbf{1 7})\end{array}$ \\
\hline Baseline AHI (events/h) & $5-14$ (8 patients) \\
& $\geq 15$ (9 patients) \\
\% nights used each month (\%) & $97.6 \pm 3.2$ \\
Hours per night, on nights APAP was used $(\mathrm{h})$ & $6.2 \pm 1.1$ \\
$\%$ nights $>4$ h per night $(\%)$ & $85.4 \pm 14.5$ \\
Minimum pressure $(\mathrm{mmHg})$ & $6.8 \pm 1.65$ \\
Maximum pressure $(\mathrm{mmHg})$ & $13.86 \pm 2.7$ \\
Pressure P95 (mmHg) & $10.5 \pm 1.7$ \\
Residual AHI (events/h) & $2.3 \pm 3.3$ \\
\hline
\end{tabular}

All data are given as the mean $\pm S D$. APAP, auto-adjusting positive airway pressure; $A H I$, apnoea-hypopnoea index.

${ }^{*}$ Of the 25 patients eligible for treatment: 4 refused treatment, 2 died before initiating treatment, and 2 abandoned the treatment in the first week.

\section{TABLE 3}

\section{Comparison between patients with and without OSA}

\begin{tabular}{|c|c|c|c|}
\hline Variables (units) & Patients without OSA $(n=15)$ & Patients with OSA $(n=34)$ & $p$ \\
\hline Age (years) & $62.5 \pm 13.4$ & $69.2 \pm 11.2$ & NS \\
\hline Gender (male/female) & $10(66.7) / 5(33.3)$ & $16(47.1) / 18(52.9)$ & NS \\
\hline \multicolumn{4}{|l|}{ Smoking history } \\
\hline Current smokers & $2(13.3)$ & $2(5.9)$ & NS \\
\hline Ex-smokers & $6(40.0)$ & $10(29.4)$ & NS \\
\hline Non-smokers & $7(46.7$ & $22(64.7)$ & NS \\
\hline \multicolumn{4}{|l|}{ Comorbidities } \\
\hline GERD & $13(86.7)$ & $21(61.8)$ & NS \\
\hline Arterial hypertension & $5(33.3)$ & $18(52.9)$ & NS \\
\hline Diabetes mellitus & $2(13.3)$ & $7(20.6)$ & NS \\
\hline Coronary disease and/or heart failure & $2(13.3)$ & $5(14.7)$ & NS \\
\hline Stroke & $1(6.7)$ & $3(8.8)$ & NS \\
\hline Pulmonary hypertension & $2 / 13(15.4)$ & $4 / 22(18.2)$ & NS \\
\hline \multicolumn{4}{|l|}{ Pulmonary function tests } \\
\hline FEV1 (\% predicted) & $77.0 \pm 20.3$ & $94.8 \pm 23.6$ & NS \\
\hline FVC (\% predicted) & $77.9 \pm 17.9$ & $89.8 \pm 18.4$ & NS \\
\hline FEV1/FVC (\%) & $77.5 \pm 9.9$ & $81.6 \pm 9.3$ & NS \\
\hline TLC (\% predicted) & $81.9 \pm 15.7$ & $85.5 \pm 14.5$ & NS \\
\hline DLCO (\% predicted) & $62.1 \pm 18.4$ & $67.5 \pm 18.8$ & NS \\
\hline \multicolumn{4}{|l|}{ Six-minute walk test } \\
\hline Distance (m) & $411.7 \pm 148.8$ & $401.8 \pm 113.9$ & NS \\
\hline $\mathrm{SpO}_{2}$ before $(\%)$ & $95.1 \pm 3.0$ & $95.7 \pm 2.2$ & NS \\
\hline $\mathrm{SpO}_{2}$ after $(\%)$ & $87.2 \pm 6.8$ & $87.5 \pm 5.8$ & NS \\
\hline \multicolumn{4}{|l|}{ Blood gas analysis } \\
\hline $\mathrm{PaO}_{2}(\mathrm{mmHg})$ & $80.8 \pm 10.7$ & $79.1 \pm 10.9$ & NS \\
\hline $\mathrm{PaCO}_{2}(\mathrm{mmHg})$ & $37.5 \pm 4.8$ & $39.2 \pm 5.3$ & NS \\
\hline Fibrosis score & $9.9 \pm 2.9$ & $8.5 \pm 3.1$ & NS \\
\hline ESS & $6.5 \pm 6.6$ & $6.6 \pm 4.6$ & NS \\
\hline$>11$ & $4(26.7)$ & $5(14.7)$ & NS \\
\hline$<11$ & $11(73.3)$ & $29(85.3)$ & NS \\
\hline \multicolumn{4}{|l|}{ Polysomnography III } \\
\hline $\mathrm{AHI}$ (events/h) & $3.0 \pm 1.4$ & $15.0 \pm 9.8$ & 0.002 \\
\hline Apnea index (events/h) & $1.2 \pm 1.4$ & $5.8 \pm 5.5$ & 0.004 \\
\hline Hypopnoea (events/h) & $1.9 \pm 1.4$ & $9.2 \pm 7.8$ & 0.020 \\
\hline ODI (events/h) & $3.4 \pm 2.1$ & $15.2 \pm 11.1$ & 0.009 \\
\hline$>10$ events/h & $0(0)$ & $23(67.6)$ & NS \\
\hline$<10$ events/h & $15(100)$ & $11(32.4)$ & NS \\
\hline Minimum $\mathrm{SpO}_{2}(\%)$ & $86.1 \pm 3.9$ & $81.5 \pm 7.6$ & NS \\
\hline Average $\mathrm{SpO}_{2}(\%)$ & $93.1 \pm 2.6$ & $92.7 \pm 3.0$ & NS \\
\hline $\mathrm{ST}<90 \%(\%)^{2}$ & $8.4 \pm 15.7$ & $16.9 \pm 27.5$ & NS \\
\hline$>20 \%$ & $2(13.3)$ & $10(29.4)$ & NS \\
\hline$<20 \%$ & $13(86.7$ & $24(70.6)$ & NS \\
\hline
\end{tabular}

All data are given as the mean $\pm S D$ or $\mathrm{n}(\%)$. OSA, obstructive sleep apnoea; NS, not statistically significant; GERD, gastro-oesophageal reflux disease; FEV1, forced expiratory volume in 1 second; FVC, forced vital capacity; TLC, total lung capacity; DLCO, diffusing capacity of lung for carbon monoxide; ESS, Epworth sleepiness score; $\mathrm{AHI}$, apnoea-hypopnoea index; ODI, oxygen desaturation index; $S T<90 \%$, sleep time with $\mathrm{SpO}{ }_{2}$ below $90 \%$. 
under APAP, and all of them presented with resolved desaturation (mean $\mathrm{SpO}_{2}: 94.3 \pm 1.6 \%$ and mean ST<90\%: $4.5 \pm 2.8 \%$ ). The most frequent complaints related to APAP treatment were sleep onset and maintenance insomnia $(n=3,17.6 \%)$ and dry mouth despite heated humidification $(n=3,17.6 \%)$.

\section{DISCUSSION}

In this study, even after excluding obesity and other risk factors for OSA, a high prevalence of this condition was found in fibrotic DPLD patients (69.4\%), mainly in IPF (83.3\%) and CHP (76.2\%) cases. In addition, there was good compliance with the APAP treatment in fibrotic DPLD patients. In this study group, OSA severity was mostly mild, as reported in some studies of DPLD patients [5, 6] but in contrast with others $[4,7,8,19]$.

Fibrotic DPLD is characterized by a decreased lung volume that can reduce the upper airway stability and increase resistance due to decreased traction in the upper airway [11-14]. Based on this information, a significant correlation was expected between AHI/OSA severity and PFTs values, such as FVC and TLC. However, we did not find significant differences in the PFTs between patients with and without OSA. Additionally, there have been conflicting reports on this subject in literature, with most studies failing to demonstrate this relationship [4-6]. The lack of an inverse correlation between PFTs and OSA severity may be explained because PFTs were obtained with the patient in a seated position and not in a supine position [4-6]. The FVC and TLC obtained with the patient in a supine position may be more accurate at predicting sleep lung volumes and may better display the interdependence between upper airway and lung volumes during sleep [4-6]. Nevertheless, other explanations can also be addressed, including the small number of patients included in these studies as well as the existence of other mechanisms increasing OSA incidence in DPLD, such as proinflammatory cytokines released due to underlying lung inflammation with subsequent oedema and swelling of the upper airway that would increase the likelihood of OSA [28].

The respiratory events were predominantly hypopnoea rather than apnoea, as observed in previous reports [4, 6]. The ODI and percentage with an ST $<90 \%$ were the parameters that exhibited greater differentiation in patients with and without OSA. Oxygen desaturation during sleep, measured as ODI [6] and minimum $\mathrm{SpO}_{2}[4,7,31]$, showed a correlation with AHI in several reports. In contrast, in this study, ESS score did not differ between patients with and without OSA, which is in agreement with previous studies [4-7, 31]. Based on these results, in the absence of other OSA screening tools in fibrotic DPLD patients and understanding that PSG is an expensive procedure to be performed in all patients, the authors suggest that nocturnal oximetry should help in recognizing those patients at risk of OSA.

OSA in fibrotic DPLD patients may be one reason for impaired sleep quality and, consequently, may have a negative influence on daily activities and overall quality of life [15-17]. In addition, underlying OSA and other SBDs such as sleep oxygen desaturation appear to have a negative influence on mortality in fibrotic DPLD patients, namely IPF [7, 8]. Recent studies have shown that effective PAP treatment (good compliance) in IPF patients with OSA resulted in a significant improvement in the daily living activities, as well as quality of sleep and life, and may also influence the mortality in these patients $[8,19]$. Therefore, OSA treatment in fibrotic DPLD patients appears to be emergent. As such, the most common difficulties in compliance should be identified, and solutions should be found to improve treatment acceptance.

There are a few studies about OSA treatment in DPLD, and all of them included only IPF patients $[8,19]$. In our study, we included other fibrotic DPLDs, namely CHP, which is common in Portugal and like IPF is often associated with a poor prognosis [32].

High incidences of nonacceptance and poor compliance to PAP in IPF patients with OSA have been reported $[8,19,20]$. The following main therapeutic difficulties have been noted [20]: frequent complaints of a nocturnal, dry, and irritating cough; discomfort and claustrophobia associated with the use of facial or oronasal masks; sleep problems such as sleep onset and maintenance insomnia; depression; and low incidence of excessive daytime sleepiness. In our study, 4 patients that were eligible for OSA treatment refused APAP therapy. The authors considered that this rate of treatment refusal was the most important problem in this group of patients. Some possible reasons for this could be related to the lack of excessive daytime sleepiness as well as the presence of other comorbidities with consequent follow-ups in multiple medical appointments. These patients may have viewed APAP treatment as just another problem rather than a solution with added benefits. This attitude could be diminished if physicians involved in the management and follow-up of fibrotic DPLD patients were sensitive to this comorbidity. At the beginning of treatment, they should explain that some of the symptoms, like daytime fatigue, could be related to OSA and clarify the potential benefits of the treatment.

Two patients started APAP treatment but reported intolerance (claustrophobia and/or insomnia) in the first week and left the treatment. In the remaining patients $(n=17)$ that started APAP treatment, the compliance was good enough to correct AHI and oxygen desaturation without supplemental oxygen therapy. Oxygen desaturation during sleep has a negative association with survival in fibrotic DPLD, namely IPF [7]. Consequently, an oxygen desaturation correction with APAP treatment may contribute to achieve better results in the survival of these patients. The good compliance in this study group was possible due to a well-organized sleep clinic that was able to deal with and to overcome difficult cases. Furthermore, APAP treatment may be more comfortable for patients than CPAP treatment as reported previously [33].

Our study had several limitations, such as the small size and heterogeneity of DPLD cases. In addition, the use of PSG III instead of PSG I could have underestimated OSA severity. Moreover, it did not provide any information about sleep architecture. On the other hand, the OSA impact and of treatment compliance in patients' quality of life and mortality is one of the most important outcomes in this population. However, in this study we had too small a sample and short follow-up period for this assessment. This evaluation should be included in future studies.

\section{CONCLUSION}

In conclusion, we found a high prevalence of OSA among patients with fibrotic DPLD, including IPF, CHP, connective-tissue associated DPLD, and stage IV sarcoidosis. The ODI and percentage of ST $<90 \%$ were the more differentiated parameters between patients with and without OSA. Therefore, nocturnal oximetry should be performed in all patients with fibrotic DPLD to determine those that require a more thorough evaluation with PSG. In patients with fibrotic DPLD and OSA, APAP treatment could be sufficient to correct oxygen desaturation. Through recognition of these comorbidities by physicians involved in the management/follow-up of fibrotic DPLD patients and their articulation with the sleep clinic, it would be possible contribute to a good APAP compliance and a possible positive impact on survival.

\section{ACKNOWLEDGEMENTS}

The authors would like to thank all co-workers from the Respiratory Sleep Disorders Unit of Pulmonology Department of Centro Hospitalar São João for their help in collecting the data for this study.

\section{REFERENCES}

1. American Thoracic Society, European Respiratory Society. American Thoracic Society/European Respiratory Society international multidisciplinary consensus classification of the idiopathic interstitial pneumonias. Am J Respir Crit Care Med 2002;165: 277-304. doi: 10.1164/ ajrccm.165.2.ats01.

2. Travis WD, Costabel U, Hansell DM, et al. An official American Thoracic Society/European Respiratory Society statement: Update of the international multidisciplinary classification of the idiopathic interstitial pneumonias. Am J Respir Crit Care Med 2013;188(6): 733-48. doi: 10.1164/rccm.201308-1483ST.

3. Raghu G, Collard HR, Egan JJ, et al. An official ATS/ERS/JRS/ALAT statement: Idiopathic pulmonary fibrosis: Evidence-based guidelines for 
diagnosis and management. Am J Respir Crit Care Med 2011;183: 788824. doi: $10.1164 /$ rccm.2009-040GL.

4. Lancaster LH, Mason WR, Parnell JA, et al. Obstructive sleep apnea is common in idiopathic pulmonary fibrosis. Chest 2009;136(3): 772-8. doi: $10.1378 /$ chest.08-2776.

5. Mermigkis C, Stagaki E, Tryfon S, et al. How common is sleep-disordered breathing in patients with idiopathic pulmonary fibrosis?. Sleep Breath 2010;14: 387-90. doi: 10.1007/s11325-010-0336-5.

6. Pithtili A, Bingol Z, Kiyan E, Cuhadaroglu C, Issever H, Gulbaran Z, Obstructive sleep apnea is common in patients with interstitial lung disease. Sleep Breath 2013;17: 1281-88. doi: 10.1007/s11325-013-0834-3.

7. Kolilekas L, Manali E, Vlami KA, et al. Sleep oxygen desaturation predicts survival in idiopathic pulmonary fibrosis. J Clin Sleep Med 2013;9(6): 593-601. doi: 10.5664/jcsm.2758.

8. Mermigkis C, Bouloukaki I, Antoniou K, et al. Obstructive sleep apnea should be treated in patients with idiopathic pulmonary fibrosis. Sleep Breath 2014;19(1): 385-91. doi: 10.1007/s11325-014-1033-6.

9. Oldham JM, Collard HR, Comorbid conditions in idiopathic pulmonary fibrosis: Recognition and management. Front Med 2017;4(123): 1-9. doi: 10.3389/fmed.2017.00123.

10. Mavroudi M, Papakosta D, Kontakiotis T, et al. Sleep disorders and health-related quality of life in patients with interstitial lung disease. Sleep Breath 2018;22(2): 393-400. doi: 10.1007/s11325-017-1579-1.

11. Aronson RM, Carley DW, Onal E, Upper airway muscle activity and the thoracic volume dependence of upper airway resistance. J Appl Physiol 1991;70: 430-8. doi: 10.1152/jappl.1991.70.1.430.

12. Sériès F, Cormier Y, Lampron N, La Forge J, Increasing the functional residual capacity may reverse obstructive sleep apnea. Sleep 1988;11: 349-53

13. Sériès F, Cormier Y, Lampron N, La Forge J, Influence of lung volume in sleep apnea. Thorax 1989;44: 52-7. doi: 10.1136/thx.44.1.52.

14. Heinzer RC, Stanchina ML, Malhotra A, et al., Lung volume and continuous positive airway pressure requirements in obstructive sleep apnea. Am J Respir Crit Care Med 2005;172: 114-17. doi: 10.1164/rccm. 200404-552OC.

15. Krishnan V, McCormack MC, Mathai SC, et al. Sleep quality and health-related quality of life in idiopathic pulmonary fibrosis. Chest 2008;134: 693-8. doi: 10.1378/chest.08-0173.

16. De Vries J, Kessels BL, Drent M, Quality of life ofidiopathic pulmonary fibrosis patients. Eur Respir J 2001;17: 954-61. doi: 10.1183/09031936. 01.17509540.

17. Swigris JJ, Kuschner WG, Jacobs SS, Wilson SR, Gould MK, Healthrelated quality of life in patients with idiopathic pulmonary fibrosis: A systematic review. Thorax 2005;60: 588-94. doi: 10.1136/thx.2004. 035220 .

18. Mermigkis C, Bouloukaki I, Schiza SE, Obstructive sleep apnea in patients with interstitial lung diseases: Past and future. Sleep Breath 2013;17: 1127-28. doi: 10.1007/s11325-013-0836-1.

19. Mermigkis C, Bouloukaki I, Antoniou K, et al. CPAP therapy in patients with idiopathic pulmonary fibrosis and obstructive sleep apnea. Does it offer a better quality of life and sleep? Sleep Breath 2013;17: 1137-43. doi: $10.1007 / \mathrm{s} 11325-013-0813-8$.

20. Mermigkis C, Mermigkis D, Varouchakis G, Schiza S, CPAP treatment in patients with idiopathic pulmonary fibrosis and obstructive sleep apnea - Therapeutic difficulties and dilemmas. Sleep Breath 2012;16: 1-3. doi: 10.1007/s11325-010-0476-7.

21. Berry RB, Budhiraia R, Gottlieb DJ, et al. Deliberations of the sleep apnea definitions Task Force of the American Academy of Sleep Medicine. Rules for scoring respiratory events in sleep: Update of the 2007 AASM manual for the scoring of sleep and associated events. J Clin Seep Med 2012;8(5): 597-619. doi: 10.5664/jcsm.2172.

22. Epstein LJ, Kristo D, Strollo PJ, Jr et al. Adult Obstructive Sleep Apnea Task Force of the American Academy of Sleep. Clinical guideline for the evaluation, management and long-term care of obstructive sleep apnea in adults. J Clin Sleep Med 2009;5(3): 263-76

23. Chung C, Liao P, Elsaid H, Islam S, Shapiro CM, Sun Y, Oxygen desaturation index from nocturnal oximetry: A sensitive and specific tool to detect sleep-disordered breathing in surgical patients. Anesth Analg 2012;114: 993-1000. doi: 10.1213/ANE.0b013e318248f4f5.

24. American Thoracic Society/European Respiratory Society Task Force. Standardisation of lung function testing. Eur Respir J 2005;26:319-38

25. Holland AE, Spruit MA, Troosters T, et al. An official European Respiratory Society/American Thoracic Society technical standard: Field walking tests in chronic respiratory disease. Eur Respir J 2014;44(6): 1428-46. doi: 10.1183/09031936.00150314.

26. Wells AU, Desai SR, Rubens MB, et al. Idiopathic pulmonary fibrosis: A composite physiologic index derived from disease extent observed by computed tomography. Am J Respir Crit Care Med 2003;167(7): 962-69. doi: 10.1164/rccm.2111053.

27. Wells AU, Rubens MB, du Bois RM, Hansell DM, Serial CT in fibrosing alveolitis: Prognostic significance of the initial pattern. Am J Roentgenol 1993;161: 1159-65. doi: 10.2214/ajr.161.6.8249719.

28. Johns MW, A new method for measuring daytime sleepiness: The Epworth sleepiness scale. Sleep 1991;14: 540-5. doi: 10.1093/sleep/ 14.6.540.

29. Van Zeller M, Severo M, Santos AC, Drummond M, 5-years APAP adherence in OSA patients - Do first impressions matter?. Respir Med 2013; 107(12): 2046-52. doi: 10.1016/j.rmed.2013.10.011.

30. Engleman HM, Wild MR, Improving CPAP use by patients with the sleep apnea/hypopnea syndrome. Sleep Med Rev 2003;7(1): 81-99. doi: 10.1053/smrv.2001.0197.

31. Romem A, Iacono A, Mcllmoyle E, et al. Obstructive sleep apnea in patients with end-stage lung disease. J Clin Sleep Med 2013;9(7): 687-93. doi: $10.5664 /$ jcsm. 2840 .

32. Costabel U, Bonella F, Guzman J, Chronic hipersensitivity pneumonitis. Clin Chest Med 2012;33: 151-63. doi: 10.1016/j.ccm.2011.12.004.

33. Shirlaw T, Hanssen K, Duce B, Hukins C, A randomized crossover trial comparing autotitrating and continuous positive airway pressure in subjects with symptoms of aerophagia: Effects on compliance and subjective symptoms. J Clin Sleep Med 2017;13(7): 881-88. doi: 10.5664/jcsm.6658. 